\title{
Adverse Events Following Posterior Lumbar Fusion: A Comparison of Spine Surgeons Perceptions and Reported Data for Rates and Risk Factors
}

\author{
NATHANIEL T. ONDECK, BS, ${ }^{1}$ DANIEL D. BOHL, MD, MPH,${ }^{2}$ PATAWUT BOVONRATWET, BS, ${ }^{1}$ \\ RYAN P. MCLYNN, BS, ${ }^{1}$ JONATHAN J. CUI, BS, ${ }^{1}$ ANDRE M. SAMUEL, MD, ${ }^{3}$ MATTHEW L. WEBB, \\ MD,${ }^{4}$ JONATHAN N. GRAUER, MD ${ }^{1}$ \\ ${ }^{I}$ Department of Orthopedics and Rehabilitation, Yale School of Medicine, New Haven, Connecticut, ${ }^{2}$ Department of Orthopedic Surgery, Rush University Medical \\ Center, Chicago, Illinois, ${ }^{3}$ Hospital for Special Surgery, New York, New York, ${ }^{4}$ Department of Orthopedic Surgery, Hospital of the University of Pennsylvania, \\ Philadelphia, Pennsylvania
}

\begin{abstract}
Background: Postoperative complications and risks factors for adverse events play an important role in both decision making and patient expectation setting. The present study serves to contrast surgeons' perceived and reported rates of postoperative adverse events following posterior lumbar fusion (PLF) and to assess the accuracy of predicting the impact of patient factors on such outcomes.

Methods: A survey investigating perceived rates of adverse events and the impact of patient risk factors on them following PLF for degenerative conditions was distributed to spine surgeons at the Lumbar Spine Research Society (LSRS) 2016 annual meeting. For comparison, the corresponding rates and patient risk factors were assessed in patients undergoing elective PLF from the American College of Surgeons National Surgical Quality Improvement Program (NSQIP) data years 2011-2014.

Results: From the survey, there were 53 responses (response rate of 79\%) from attending physicians at LSRS. From NSQIP, there were 16,589 patients who met the inclusion criteria. Adverse event rates estimated by the surgeons at LSRS were close to those determined by NSQIP data (no greater than $2.81 \%$ different). The largest differences were for deep vein thrombosis (overestimation of $2.81 \%, P<.001$ ), anemia requiring transfusion (overestimation of $2.47 \%$, $P=.018$ ), and urinary tract infection (overestimation of $2.29 \%, P<.001$ ). Similarly, the estimated impact of patient factors was similar to the data (within relative risk of 2.02). The largest differences were for current smoking (overestimation of 2.02 relative risk, $P<.001$ ), insulin dependent diabetes (overestimation of $1.36, P<.001$ ), and obesity (overestimation of $1.35, P<.001$ ).

Conclusions: The current study noted that surgeon estimates were relatively close to national numbers for estimating the adverse events and impact of patient factors on such outcomes after PLF for degenerative conditions. The estimates are roughly appropriate with a bias toward overestimation for planning and expectation setting.
\end{abstract}

Lumbar Spine

Keywords: perceived, rates, risk factors, adverse events, posterior lumbar fusion

\section{INTRODUCTION}

Posterior lumbar spine fusion (PLF) is a commonly performed procedure that is often done concurrently with decompression for the treatment of lumbar pathology, with studies demonstrating clinical improvements. ${ }^{1-3}$ The occurrence of postoperative complications following spine surgery has received much attention in the literature, as it has been shown to have serious impacts on patients' recoveries. $^{4-7}$

To facilitate the analysis of large populations for relatively rare complications, much research has turned to national databases, including the American College of Surgeons National Surgical Quality Improvement Program (NSQIP). NSQIP collects data from over 500 hospitals across the United States and has recently been extensively used in orthopedic research. ${ }^{8-12}$ Although such studies are limited with regard to spine-specific outcome measures, they are robust for general health adverse events.

The specific affects of adverse events on patient's recoveries following spine surgery has been demonstrated with both large national data sets as well as smaller cohort studies. For example, a study 
utilizing NSQIP found that wound complications, pulmonary embolism, deep vein thrombosis, sepsis, and urinary tract infections (UTIs) are all associated with unplanned hospital readmission following PLF. $^{4}$ Using a smaller sample of 155 patients from a Food and Drug Administration-approved randomized trial regarding interventions for lumbar disc degeneration, it was discovered that patients who had adverse events related to their surgery reported higher pain scores and had decreased function at 2-year follow-up in comparison those who had no adverse events. ${ }^{5}$

Furthermore, certain patient characteristics have been demonstrated to have an impact on patient recovery following elective PLF in both national data set studies as well as local cohort investigations. For instance, NSQIP studies have shown that malnutrition, increasing age, morbid obesity, and multilevel procedures are all patient factors that are associated with increased rates of adverse events and increased hospital length of stay after PLF. ${ }^{9,12}$ Additionally, a study of 1137 consecutive spinal surgeries at a single institution revealed that patients who have 2 or more comorbidities or who are having their procedure on multiple spinal levels, are more likely to be readmitted. ${ }^{13}$

Communication of risk factors for and the likelihood of adverse events to patients is important for risk stratification, surgical decision making, and patient expectation setting. In fact, the value of setting realistic expectations was demonstrated through a study of patients who underwent lumbar decompression. The investigators found that the expectation-actuality discrepancy predicted patient satisfaction regardless of the magnitude of expectations. ${ }^{14}$ Therefore, it is important that surgeons and patients have discussions that reflect accurate estimations of complication rates and true assessments of an individual's risk factors for adverse events.

To our knowledge, no recent study has compared surgeons' perception of the rates of postoperative general health adverse events and the impact of patient factors on these rates and the reported data. As this plays an important role in setting patient expectations, the purpose of the present study is to delineate these perceptions for those at the Lumbar Spine Research Society (LSRS) 2016 Annual Meeting and compare the results to those reported in NSQIP in order to gain an understanding of potential discrepancies between surgeon impressions and reported data. The hypothesis is that spine surgeons would significantly underestimate the rates of and risk factors for adverse events.

\section{MATERIALS AND METHODS}

\section{Survey}

A 1-page survey was developed and optimized to characterize the perceived rates of general health postoperative adverse events within 30 days of surgery for patients undergoing PLF procedures for degenerative conditions. Adverse events asked about, which were also recorded in NSQIP for later comparison, included: acute kidney injury, anemia requiring transfusion, cardiac arrest, coma, death, deep vein thrombosis, hospital readmission, myocardial infarction, pneumonia, postoperative intubation, pulmonary embolism, return to the operating room, sepsis, surgical site infection, stroke, UTI, and wound dehiscence.

Additionally, the survey addressed the perceived change in risk for having any of the aforementioned adverse events when certain patient factors were present compared to when they were not present. The patient factors included: chronic obstructive pulmonary disease (COPD), current smoking, dyspnea on exertion, hypertension, insulin-dependent diabetes, more than one-level surgery, noninsulin-dependent diabetes, obesity (body mass index $[\mathrm{BMI}] \geq 30$ ), older age (70 years or older), and preoperative anemia.

The survey also asked about respondent demographic information including: type of residency, completion of spine surgery fellowship, practice setting (private, academic, hybrid), practice location, and years in practice. The survey can be viewed in the appendix.

The survey was distributed to attendees at the 2016 LSRS 9th Annual Meeting. The LSRS is a society founded in 2008, whose purpose is to create an environment for discourse, free from bias, regarding lumbar disease. ${ }^{7}$ Surveys were collected following completion.

\section{NSQIP Sample}

A cohort of patients matching those asked about in the above-described survey was identified in NSQIP 2011-2014. NSQIP is a data set that collects information on over 150 variables, with 30-day follow-up, from patients undergoing surgery at over 500 sites across the nation. The data undergoes rigorous accuracy audits with combined results of audits indicating approximately $2 \%$ disagreement 
Table 1. 2016 Lumbar Spine Research Society Annual Meeting survey respondent characteristics (79\% response rate).

\begin{tabular}{lc}
\hline & Percent of Respondents \\
\hline Residency training & \\
Orthopedics & 84 \\
Neurosurgery & 16 \\
Spine fellowship training & \\
Yes & 96 \\
No & 4 \\
Practice setting & 18 \\
Private & 63 \\
Academic & 19 \\
Hybrid & \\
Geographic location & 47 \\
Midwest & 18 \\
Northeast & 19 \\
West & 16 \\
South & \\
\hline
\end{tabular}

between the data set and patient charts. ${ }^{8}$ Patients were identified using current procedural terminology codes for PLF (22612, 22630, 22633). Exclusion criteria included a primary diagnosis of fracture, neoplasm or infection, as well as patients undergoing emergency surgery, anterior/PLF, or more than 3 levels of fusion.

Patient baseline characteristics extracted from the data set included patient age, gender, BMI, and the American Society of Anesthesiologists (ASA) Classification. Using this cohort, the reported rates of the adverse events listed in the LSRS survey were extracted as well as were the patient risk factors.

\section{STATISTICAL METHODS}

For the LSRS survey data, the mean estimation for the rates of each adverse event as well as the average perceived impact of patient risk factors were calculated. For the NSQIP data, the reported rates of each adverse event were assessed and the impact of patient factors was analyzed using a Poisson regression with robust error variance. The perceived rates of adverse events and the impacts of patient factors had on these from the LSRS data were compared with the reported NSQIP data using Welch's $t$-test.

Questionnaire and NSQIP data were analyzed using STATA version 11.0 (StataCorp, LP, College Station, Texas). Statistical difference was established at a 2 -sided $\alpha$ level of $0.05(P<.05)$.

\section{RESULTS}

\section{LSRS Respondent and NSQIP Sample Demographics}

For the LSRS surveys, of the 67 United States attending-level physicians in attendance (medical
Table 2. Patient characteristics in National Surgical Quality Improvement Program years 2011-2014 for patients undergoing posterior lumbar fusion.

\begin{tabular}{lr}
\hline Patients, no. & 16589 \\
Age $(60 \pm 13.5), \mathrm{y}^{*}$ & \\
$18-29(\%)$ & 1.89 \\
$30-39(\%)$ & 6.46 \\
$40-49(\%)$ & 13.18 \\
$50-59(\%)$ & 23.93 \\
$60-69(\%)$ & 28.05 \\
$70-79(\%)$ & 21.32 \\
$80-89(\%)$ & 4.98 \\
$90+(\%)$ & 0.19 \\
Male gender $(\%)$ & 44.55 \\
Body mass index $(31 \pm 6.7), \mathrm{kg} / \mathrm{m}^{2 *}$ & \\
$<25(\%)$ & 18.55 \\
25-30 (\%) & 32.95 \\
30-35 (\%) & 26.45 \\
$>35(\%)$ & 22.06 \\
ASA classification $($ median $=2, \mathrm{IQR}=2-3)$ & \\
1-No disturbance $(\%)$ & 2.8 \\
2-Mild disturbance $(\%)$ & 48.63 \\
3-Severe disturbance $(\%)$ & 46.24 \\
4-Life threat $(\%)$ & 2.13 \\
5-Moribund $(\%)$ & 0.01 \\
\hline
\end{tabular}

*Mean \pm standard deviation.

doctors and doctors of osteopathic medicine), 53 returned completed surveys (equating to a completion rate of $79 \%$ ). The demographics of the 53 attending-level spine surgeons practicing in the United States are presented in Table 1. The LSRS questionnaire respondents were predominantly orthopedic surgeons practicing in an academic setting in the Midwest.

For the NSQIP cohort, 16,589 patients were identified based on the defined inclusion/exclusion criteria. The demographics of the identified population are presented in Table 2. The population had an average age of $60,45 \%$ of patients were male, and had an average BMI of 31 and a median ASA of 2 .

\section{Rates of Adverse Events}

The perceived incidence of the 17 adverse events from the surgeons at the conference and the reported incidence of the same adverse events from the NSQIP data can be found in the left half of Figure 1. From the LSRS survey, adverse events were estimated to range from $0.18 \%$ to $6.91 \%$. From the NSQIP cohort, adverse events were estimated to range from $0.01 \%$ to $5.22 \%$.

The differences between the LSRS survey and the NSQIP cohort results are shown graphically in the right half of Figure 1. The differences ranged from LSRS survey overestimates of $2.81 \%$ to underestimates of $1.05 \%$. Following statistical analysis, surgeons overestimated the incidence of $76 \%$ (13 

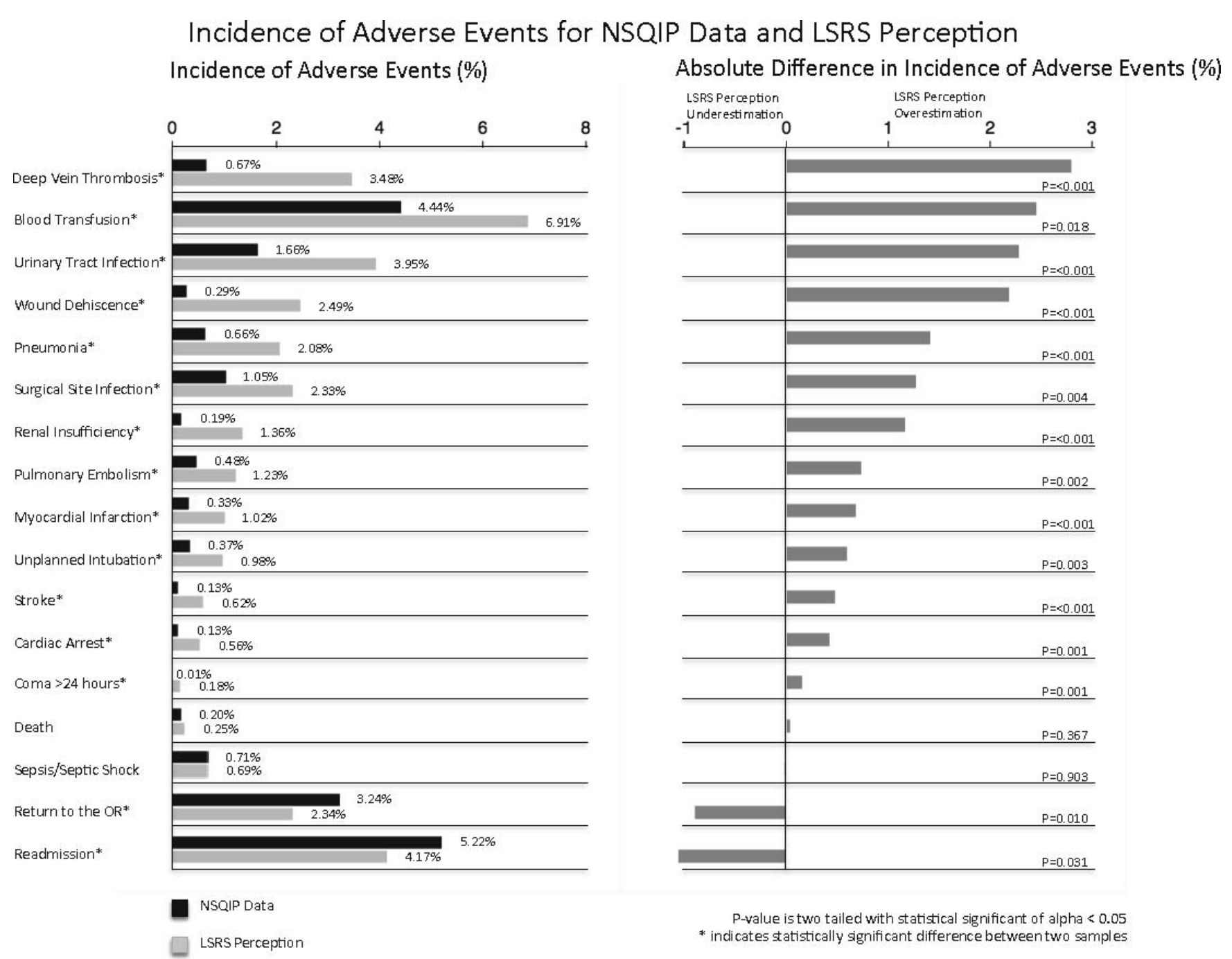

Figure 1. Incidence of adverse events for National Surgical Quality Improvement Program (NSQIP) data and Lumbar Spine Research Society (LSRS) perception.

out of 17) of the adverse events and underestimated the incidence of $12 \%$ of the adverse events ( 2 out of 17). The adverse events with the largest survey overestimations included the incidence of deep vein thrombosis $(2.81 \%, P<.001)$, anemia requiring transfusion $(2.47 \%, P=.018)$, and UTI $(2.29 \%, P$ $<.001)$. Surgeons accurately estimated the occurrence of death and sepsis following PLF with the difference in the 2 incidences being statistically insignificant. The rates of patients returning to the operating room and hospital readmission were underestimated by $0.9 \%(P=.010)$ and $1.05 \%(P$ $=.031)$, respectively.

\section{Risk Factors for Adverse Events}

The perceived impact of 10 patient characteristics on the risk of adverse events from the LSRS surgeons and the reported impact of the same patient characteristics on the risks of adverse event from the NSQIP data can be found in the left half of Figure 2. From the LSRS survey, relative risks were estimated to range from 1.19 to 2.98. From the NSQIP cohort, relative risks were estimated to range from 0.96 to 2.12 .

The differences between the LSRS survey and the NSQIP cohort results are shown graphically in the right half of Figure 2. The differences ranged from LSRS survey overestimates of 2.02 relative risk to underestimates of 0.31 relative risk. Following statistical analysis, surgeons overestimated the relative risk of $80 \%$ ( 8 out of 10 ) of the patient characteristics. The largest differences existed in patients who are current smoking (overestimation of 2.02 relative risk, $P<.001$ ), who have insulin dependent diabetics (overestimation of 1.36 relative risk, $P<.001$ ), and who are obese (overestimation of 1.35 relative risk, $P<.001$ ). Surgeons accurately 


\section{Risk Factors for Adverse Events for NSQIP Data and LSRS Perception Relative Risk of Adverse Events Absolute Difference of Relative Risk}
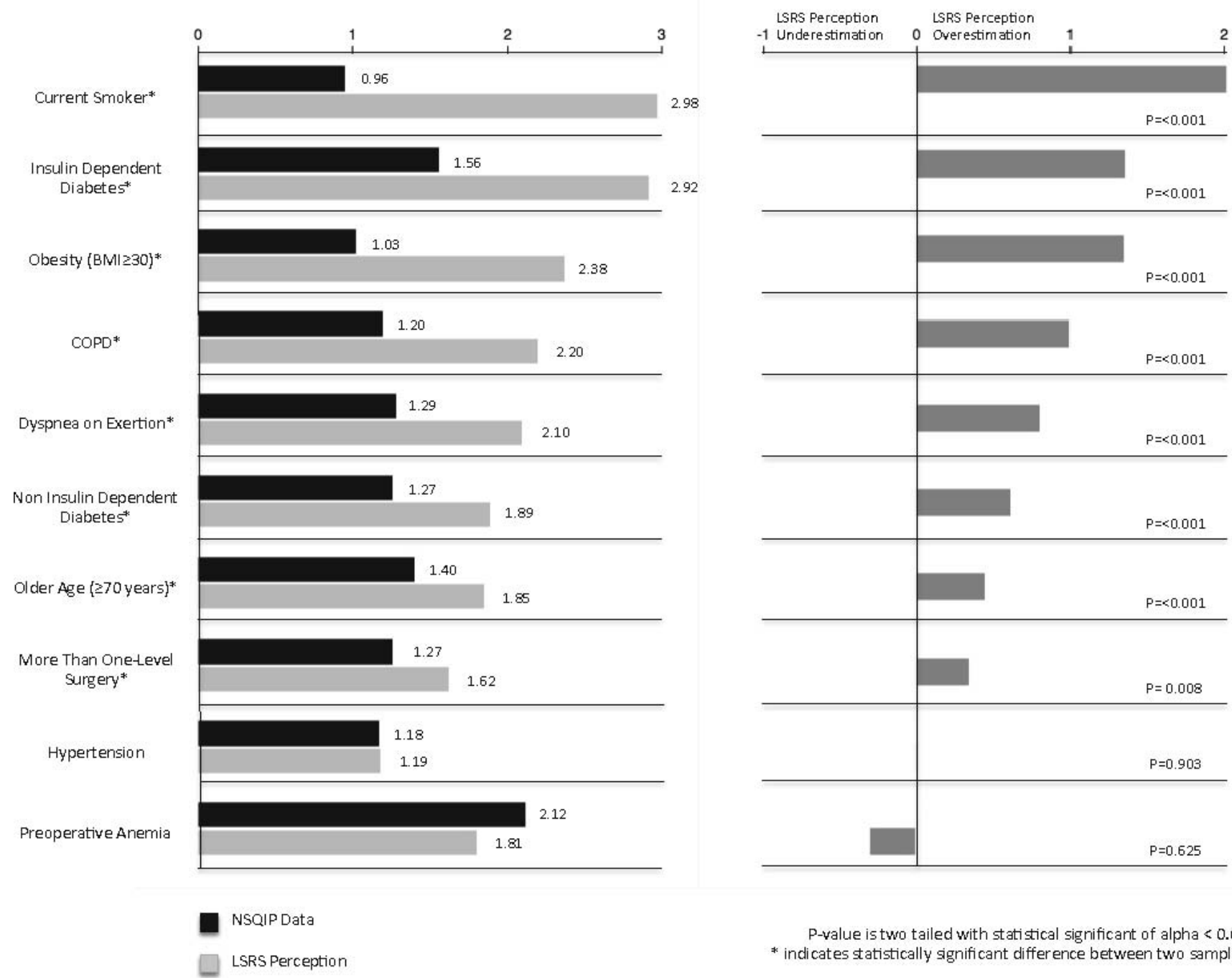

P-yalue is two tailed with statistical significant of alpha $<0.05$ * indicates statistically significant difference between two samples

Figure 2. Risk factors for adverse events for National Surgical Quality Improvement Program (NSQIP) data and Lumbar Spine Research Society (LSRS) perception.

estimated the relative risk of hypertension and preoperative anemia following PLF with the difference in the 2 incidences being statistically insignificant.

\section{DISCUSSION}

Adverse events after surgeries such as PLFs have been receiving increasing attention as we strive to minimize morbidity of such interventions and optimize outcomes. The first portion of the current study serves to contrast surgeons' perceived and NSQIP reported rates of postoperative adverse events following PLF for degenerative conditions. The second portion of the study then compares surgeons' perceived impact of patient factors on the risks for any of the previously defined adverse events with those determined from NSQIP.

The LSRS Annual Meeting attracts spine surgeons from across the country and promotes scientific discussion of the lumbar spine that is not influenced by corporate funding. ${ }^{7}$ Survey data collected at the most current meeting in April 2016 yielded a high response rate of nearly $80 \%$. This rate compares very favorably to other studies of physician surveys reported in the literature. ${ }^{15,16}$ The NSQIP database, founded in 2005, currently collects data from over 500 institutions, which include academic, private, urban, and rural settings, in the United States. ${ }^{8}$

After statistical analysis of the survey data collected at LSRS and data extracted from the 
NSQIP database, it is clear that, contrary to the hypothesis of consistent underestimation, surgeons' perceived rates of adverse events following PLF for degenerative diseases were overall in similar range to the NSQIP data. Surgeons marginally overestimated the incidence of $76 \%$ (13 out of 17 ) of the adverse events and only slightly underestimated $12 \%$ ( 2 out of 17) (Figure 1). Although these were in the right "ballpark," the numerically greatest overestimations are discussed.

The $2.81 \%$ overestimation of deep-vein thrombosis may be due surgeons' knowledge of higher rates of venous thromboembolism in other orthopedic surgeries and the subsequent transference of that information to spine procedures. For example, a recent study found the incidence of symptomatic venous thromboembolism following total knee arthroplasty to be $4.6 \% .^{17}$

The $2.47 \%$ overestimation of the rates of postoperative anemia requiring transfusion may in part be due to the development and use of minimally invasive spine surgery in cases where an open approach may have been traditionally the only option. A recent meta-analysis found there to be a mean decrease in estimated blood loss of $260 \mathrm{~mL}$ when comparing minimally invasive approaches with open surgeries. ${ }^{18}$

Finally, the $2.29 \%$ overestimation of the incidence of UTIs may be due to recent advancements in the understanding of risk factors for these infections as well as the identification of helpful preventive measures such as early removal of Foley catheters. $^{19}$

In opposition of the hypothesized consistent underestimation, surgeons' perceived impact of patient factors on the risks for any of the previously defined adverse events were also reasonably close to the NSQIP data. Surgeons modestly overestimated the relative risk of $80 \%$ ( 8 out of 10 ) of the patient characteristics while underestimating none (Figure 2).

The magnitude that current smoking has on the occurrence of adverse events had a 2.02 overestimation by spine surgeons, which may in part be due to transference of the affect that smoking has on longer term nonunion to the general-health outcomes analyzed in the current study. ${ }^{20}$ The overestimation of the association of insulin-dependent diabetes (1.36) and obesity (1.35) with the occurrence of adverse events could be related to recent focuses in the literature on the effects of these modifiable risk factors. ${ }^{10,21,22}$

Understanding and accurately estimating the risks of surgery are critical for both surgeons and patients in the decision-making process and informed consent. Patients need to have a thorough understanding of the potential risks of surgery in order to have informed consent while surgeons need accurate information regarding surgical risks in order to choose the right operation. ${ }^{23}$ Furthermore, research has indicated that quality preoperative information facilitates patient's active involvement in his or her own care. ${ }^{24}$ While the error in surgeon perceived occurrences and risks of adverse events in this study can be characterized as overestimation, this type of overemphasis benefits the safety of the patient in terms of their understanding of the risks of the procedure and expectations regarding a potentially complicated postoperative recovery. Additionally, the findings of the present study encourage physicians to continue to remain up-to-date on the current rates and risk factors for postoperative complications, especially as surgical techniques improve, postoperative management evolves, and patient populations change.

Strengths of this investigation include the novelty of the research question. To the authors' knowledge, no recent study has compared surgeons' understanding of the perceived rates of postoperative adverse events and the apparent impact of patient factors on the rates of those adverse events with the reported data. Another strength of the study is the very high survey participation rate and the quality of data that NSQIP provides. ${ }^{25}$ Limitations of the current study are that only 1 specific spine society was surveyed, which may lead to sampling bias. In particular, the LSRS surgeons may have practice type or geographical location discrepancies in comparison with the physicians captured by NSQIP. As Table 1 demonstrates, surveyed physicians were primarily from the United States Midwest (although there was some representation from all areas of the United States) and were largely practicing in an academic setting (although some private and hybrid setting physicians were captured). In addition, the association of hospital size and complication rates is not entirely clear, and, in fact, it has been suggested that there may be none following PLF. $^{26}$ A third limitation is that NSQIP only 
collects short-term adverse events no longer than the 30th postoperative day. ${ }^{27}$ Further, the current study does not report procedure-specific complications.

In summary, spine surgeons in the United States reasonably estimate the occurrence of postoperative adverse events as well as the impact of risk factors on those adverse events for patients who underwent PLF for degenerative diseases. The consistent overestimation was marginal and not only errs on the side of patient safety when it comes to decision making but also sets realistic expectations for the postoperative course.

\section{REFERENCES}

1. Fritzell P, Hagg O, Wessberg P, Nordwall A. 2001 Volvo Award Winner in Clinical Studies: lumbar fusion versus nonsurgical treatment for chronic low back pain: a multicenter randomized controlled trial from the Swedish Lumbar Spine Study Group. Spine (Phila Pa 1976). 2001;26(23):2521-2532; discussion 2532-2524.

2. Mirza SK, Deyo RA, Heagerty PJ, Turner JA, Martin BI, Comstock BA. One-year outcomes of surgical versus nonsurgical treatments for discogenic back pain: a community-based prospective cohort study. Spine J. 2013;13(11):1421-1433.

3. Zucherman JF, Hsu KY, Hartjen CA, et al. A multicenter, prospective, randomized trial evaluating the X STOP interspinous process decompression system for the treatment of neurogenic intermittent claudication: two-year follow-up results. Spine (Phila Pa 1976). 2005;30(12):1351-1358.

4. Lee NJ, Kothari P, Phan K, et al. The incidence and risk factors for 30-day unplanned readmissions after elective posterior lumbar fusion. Spine (Phila Pa 1976). 2018;43(1):41-48.

5. Ohnmeiss DD, Bodemer W, Zigler JE. Effect of adverse events on low back surgery outcome: twenty-four-month follow-up results from a Food And Drug Administration investigational device exemptiontrial. Spine (Phila Pa 1976). 2010;35(7):835-838.

6. Yadla S, Ghobrial GM, Campbell PG, et al. Identification of complications that have a significant effect on length of stay after spine surgery and predictive value of 90-day readmission rate. J Neurosurg Spine. 2015;23(6):807-811.

7. Culler SD, Jevsevar DS, Shea KG, et al. The incremental hospital cost and length-of-stay associated with treating adverse events among medicare beneficiaries undergoing lumbar spinal fusion during fiscal year 2013. Spine (Phila Pa 1976). 2016;41(20):1613-1620.

8. User Guide for the 2014 ACS NSQIP Participant Use File, in: American College of Surgeons National Surgical Quality Improvement Program. Chicago, IL: American College of Surgeons, 2015.

9. Basques BA, Fu MC, Buerba RA, Bohl DD, Golinvaux NS, Grauer JN. Using the ACS-NSQIP to identify factors affecting hospital length of stay after elective posterior lumbar fusion. Spine (Phila Pa 1976). 2014;39(6):497-502.

10. Basques BA, Ibe I, Samuel AM, et al. Predicting postoperative morbidity and readmission for revision posterior lumbar fusion. Clin Spine Surg. 2017;30(6):E770-E775.

11. Bohl DD, Ahn J, Tabaraee E, et al. Urinary tract infection following posterior lumbar fusion procedures: an American College of Surgeons National Surgical Quality Improvement Program Study. Spine (Phila Pa 1976). 2015;40(22):1785-1791.

12. Bohl DD, Shen MR, Mayo BC, et al. Malnutrition predicts infectious and wound complications following posterior lumbar spinal fusion. Spine (Phila Pa 1976). 2016;41(21):16931699.

13. Khanna R, McDevitt JL, McClendon J Jr, Smith ZA, Dahdaleh NS, Fessler RG. Utility of readmission rates as a quality of care measure and predictors of readmission within 30 days after spinal surgery: a single-center, multivariate analysis. Spine (Phila Pa 1976). 2015;40(22):1769-1774.

14. Mannion AF, Junge A, Elfering A, Dvorak J, Porchet F, Grob D. Great expectations: really the novel predictor of outcome after spinal surgery? Spine (Phila Pa 1976). 2009;34(15):1590 1599.

15. Asch DA, Jedrziewski MK, Christakis NA. Response rates to mail surveys published in medical journals. $J$ Clin Epidemiol. 1997;50(10):1129-1136.

16. Cummings SM, Savitz LA, Konrad TR. Reported response rates to mailed physician questionnaires. Health Serv Res. 2001;35(6):1347-1355.

17. Song K, Xu Z, Rong Z, et al. The incidence of venous thromboembolism following total knee arthroplasty: a prospective study by using computed tomographic pulmonary angiography in combination with bilateral lower limb venography. Blood Coagul Fibrinolysis. 2016;27(3):266-269.

18. Goldstein CL, Macwan K, Sundararajan K, Rampersaud YR. Perioperative outcomes and adverse events of minimally invasive versus open posterior lumbar fusion: meta-analysis and systematic review. J Neurosurg Spine. 2016;24(3):416-427.

19. Stephan F, Sax H, Wachsmuth M, Hoffmeyer P, Clergue F, Pittet D. Reduction of urinary tract infection and antibiotic use after surgery: a controlled, prospective, before-after intervention study. Clin Infect Dis. 2006;42(11):1544-1551.

20. Andersen T, Christensen FB, Laursen M, Hoy K, Hansen ES, Bunger C. Smoking as a predictor of negative outcome in lumbar spinal fusion. Spine (Phila Pa 1976). 2001;26(23):2623-2628.

21. Qin C, Kim JY, Hsu WK. Impact of insulin dependence on lumbar surgery outcomes: an NSQIP analysis of 51,277 patients. Spine (Phila Pa 1976). 2016;41(11):E687-E693.

22. Owens RK 2nd, Djurasovic M, Onyekwelu I, Bratcher KR, McGraw KE, Carreon LY. Outcomes and revision rates in normal, overweight, and obese patients 5 years after lumbar fusion. Spine J. 2016;16(10):1178-1183.

23. Bilimoria KY, Liu Y, Paruch JL, et al. Development and evaluation of the universal ACS NSQIP surgical risk calculator: a decision aid and informed consent tool for patients and surgeons. J Am Coll Surg. 2013;217(5):833-842.

24. Walker JA. What is the effect of preoperative information on patient satisfaction? Br J Nurs. 2007;16(1):27-32.

25. Schilling PL, Hallstrom BR, Birkmeyer JD, Carpenter JE. Prioritizing perioperative quality improvement in orthopaedic surgery. J Bone Joint Surg Am. 2010;92(9):1884-1889.

26. Kalanithi PS, Patil CG, Boakye M. National complication rates and disposition after posterior lumbar fusion for acquired spondylolisthesis. Spine (Phila Pa 1976). 2009;34(18):1963-1969. 
27. Khuri SF. The NSQIP: a new frontier in surgery. Surgery. 2005;138(5):837-843.

Disclosures and COI: The authors received no funding for this study and report no conflicts of interest. The institutional review board at the senior author's institution approved this study as an exemption.

Corresponding Author: Jonathan N. Grauer, MD, Department of Orthopedics and Rehabilita- tion, Yale School of Medicine, 800 Howard Ave, New Haven, CT 06510. Phone: (203) 737-7463; Fax: (203) 785-7132; Email: jonathan.grauer@yale.edu.

Published 15 October 2018

This manuscript is generously published free of charge by ISASS, the International Society for the Advancement of Spine Surgery. Copyright (C) 2018 ISASS. To see more or order reprints or permissions, see http://ijssurgery.com. 